\title{
Light Ion Beams Interacting with Thin Films
}

\author{
Deying $\mathrm{Xia}^{1}$ and John Notte ${ }^{1 *}$ \\ ${ }^{1 .}$ Carl Zeiss SMT, Inc., PCS Integration Center, Peabody, MA, USA. \\ * Corresponding author: John.Notte@Zeiss.com
}

Both the helium and neon ion beams are relatively light compared to gallium, and these beams are finding new applications that involve many 2D materials and thin films. For example, the helium beam has been used to precisely sputter patterns in thin films to make nanoscale plasmonic resonators in gold [1] and nanopores in silicon nitride [2]. Graphene devices have been functionalized by helium to induced lattice defects to create novel band gap devices [3]. The helium beam can also disrupt the structure of a superconducting material to create Josephson junctions for sensors or other devices [4]. Even for basic imaging tasks, the helium beam is highly regarded for its ability to make visible thin films such as self-assembled monolayers [5].

The traditional FIB beam, gallium, has recently been used to cut thin films, and then, with appropriate dosage, to cause them to bend into out-of-plane 3D shapes [6,7]. Such techniques mimic the paper cutting and folding art of kirigami, but at a much smaller scale and with precision of $\sim 50 \mathrm{~nm}$. The films that have been reported are gold, aluminum, and silicon nitride. Possible applications include making three dimensional devices such as plasmonic resonators sensitive to both direction and polarization of incident light.

In this paper we follow the previous researchers, and investigate the prospects of using the Zeiss ORION NanoFab to make similar kirigami structures with its various ion beams ( $\mathrm{Ga}, \mathrm{Ne}$, and $\mathrm{He}$ ) and various thin films. All films were commercially available on supporting grids, and all exposures were targeted for the regions where the film was freely suspended. The first sample, a formvar film (thickness 30 to 60 $\mathrm{nm}$ ) exhibited very inconsistent results, with some instances in which the formvar film failed catastrophically, quite some distance from the incident beam as though through an accumulation of stress, by heat or charge.

The testing continued with silicon nitride films, which showed more consistent results. The chosen pattern consisted of three sides of a rectangle each about 1 micron in length. The remaining edge of the rectangle was uncut, allowing for subsequent bending. With the $15 \mathrm{~nm}$ thick silicon nitride film, and using a the $30 \mathrm{keV} \mathrm{He}$ beam, the membrane was effectively cut through with a line dose, $\mathrm{D}_{1}>5 \times 10^{8}$ ions $/ \mathrm{cm}$ (estimated areal dose, $\mathrm{D}_{2}>5 \times 10^{15}$ ions $/ \mathrm{cm}^{2}$ ). Higher dosages caused wider cuts, and tended to cause the flap to bend down (away from the beam). A subsequent exposure of the whole area with the $30 \mathrm{keV}$ Ga beam with an areal dose, $\mathrm{D}_{2}>9.4 \times 10^{14}$ ions $/ \mathrm{cm}^{2}$ caused the flaps to bend further down.

For a $50 \mathrm{~nm}$ thick silicon nitride membrane, and using the same $30 \mathrm{keV} \mathrm{He}$ beam, a complete cut required a linear dosage, $\mathrm{D}_{1}>1.5 \times 10^{9}$ ions $/ \mathrm{cm}$ (estimated areal dose, $\mathrm{D}_{2}>1.6 \times 10^{16}$ ions $/ \mathrm{cm}^{2}$ ). Higher dosages caused the cuts to grow wider, and possibly to induce some slight bending. Subsequent broad exposure by the $30 \mathrm{keV}$ gallium beam caused a progressive upward bending of the flap as the areal dose, $\mathrm{D}_{2}$, increased from $6 \times 10^{14}$ to $1.2 \times 10^{15}$ ions $/ \mathrm{cm}^{2}$. Figure 1 shows the typical results of the $50 \mathrm{~nm}$ silicon nitride film after helium cutting, gallium bending, and then tilting for better examination with the helium beam. 


\section{References:}

[1] M Semple, E Baladi, and AK Iyer, IEEE Selected Topics in Quantum Electronics (2019) (accepted for publication).

[2] Xia et al., Nanoscale 10 (2018), p. 5198.

[3] Nakaharai, et al., IEEE Tansactions on Nanotechnology 13 (2014), p. 1039.

[4] L Kasaei et al., AIP Advances 8 (2018), p. 075020.

[5] A Beyer and Beilstein, J. Nanotechnol. 6 (2015), p. 1712.

[6] WJ Arora et al., J. Vac. Sci. Technol. B 25 (2007), p. 2184.

[7] Z Liu et. al., Sci. Adv. 4 (2018), p. eeat4436.
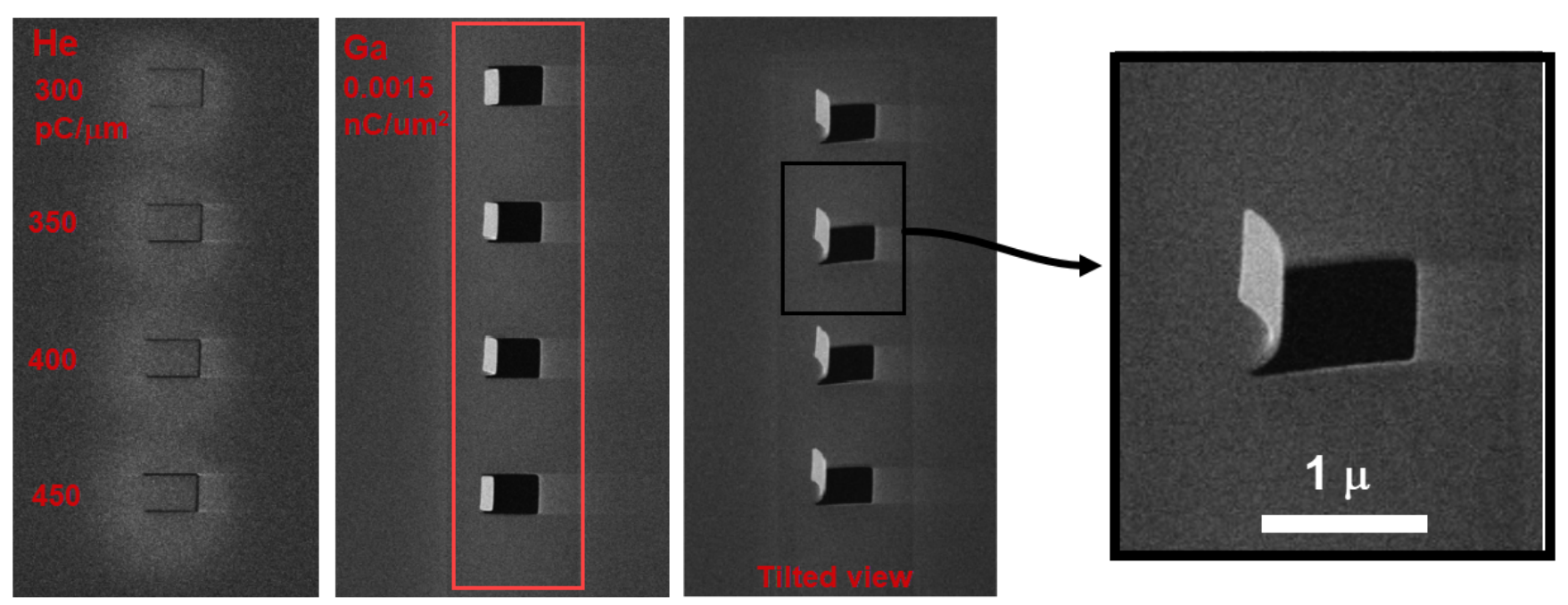

Figure 1. (left to right) A $50 \mathrm{~nm}$ thick silicon nitride flap is first cut with the helium beam at various line dosages as indicated. Then the entire region is exposed with the indicated gallium dosage. The results is inspected after tilting the sample. 